\title{
Exponentially Weighted Particle Filter for Simultaneous Localization and Mapping Based on Magnetic Field Measurements
}

\author{
Xinheng Wang, Senior Member, IEEE, Congcong Zhang, Fuyu Liu, Yuning Dong, Member, IEEE, \\ and Xiaolong Xu Member, IEEE,
}

\begin{abstract}
This paper presents a simultaneous localization and mapping (SLAM) method that utilizes the measurement of ambient magnetic fields present in all indoor environments. In this paper, an improved exponentially weighted particle filter was proposed to estimate the pose distribution of the object and a Kriging interpolation method was introduced to update the map of the magnetic fields. The performance and effectiveness of the proposed algorithms were evaluated by simulations on Matlab based on a map with magnetic fields measured manually in an indoor environment and also by tests on the mobile devices in the same area. From the tests, two interesting phenomena have been discovered; one is the shift of location estimation after sharp turning, the other is the accumulated errors. Whist the latter has been confirmed and investigated by a few researchers, the reason for the first one still remains unknown. The tests also confirm that the interpolated map by using the proposed method improves the localization accuracy.
\end{abstract}

Index Terms-Magnetic localisation, particle filter, Kriging interpolation, simultaneous localization and mapping (SLAM)

\section{INTRODUCTION}

M AGNETIC fields have been used as a means for navigation by a variety of animals [1]. However, how the animals use the magnetic field to navigate remains a mystery until recently. In a paper published recently [2], scientists discovered, by analyzing 56 years of fishery data, that sockeye salmons used the variation of the geomagnetic fields, particularly the variation of magnetic intensity and inclination angle, to navigate themselves home. The variations of the geomagnetic fields are imprinted in their body as a map and this map-sensing ability helps them home. This hypothesis is supported by other scientists in their experimental studies [3].

The ability of applying the variation of the magnetic fields in navigation has been echoed in indoor location and navigation by researchers in [4]. In that study it revealed that the magnetic

Manuscript received July 29, 2016. This work was supported in part by Wuxi Chigoo Interactive Technology Co. Ltd, National Natural Science Foundation of China under Grants 61271233 and 61472192, and the Scientific and Technological Support Project (Society) of Jiangsu Province under Grant BE2016776.

Xinheng Wang is with School of Engineering and Computing, University of the West of Scotland, Paisley, PA1 2BE, Scotland (email:xinheng.wang@uws.ac.uk).

Congcong Zhang is with Alcatel-Lucent Shanghai Bell Co., Ltd, Nanjing, China(e-mail:congcong.a.zhang@alcatel-sbell.com.cn).

Fuyu Liu, Yuning Dong, and Xiaolong $\mathrm{Xu}$ are with Nanjing University of Posts and Telecommunications, Nanjing, China (e-mail 15850790089@163.com,dongyn@njupt.edu.cn, xuxl@njupt.edu.cn). fields generated in indoor environment were rather stable and repeatable. The pattern and the signature of the magnetic fields could be used for indoor location and navigation. The idea of applying magnetic field for indoor location and navigation has been confirmed by other scientists [5] in a study of navigating a robot in a corridor and the research was extended further by other researchers to a two-dimensional space [6-7] and three-dimensional space [8], which is closer to the applications in practice. In both studies, a map-based solution was also applied to find the right location. Map-based solution, also referred to fingerprinting, is possibly the only solution to solve this kind of localization problem because of the fluctuations of the measured magnetic fields, as demonstrated in [5-6].

Fingerprinting is a popular approach in solving localization problem based on the measurement of unsteady signals, such as WiFi- and ZigBee-based localization. To implement a fingerprinting approach, a database has to be established to store the measurements and then newly measured data is used to match the measurements in the database. The best possible match between the newly measured data and stored data in the database is supposed to be the location. Kalman filter and particle filter are two popular methods used in matching. For example in [5-8] a particle filter was used to solve the localization problem.

However, to establish a database for a large-scale space, such as an airport, a hospital, or a shopping mall, is not an easy job. To solve this problem, Simultaneous Localization And Mapping (SLAM) was developed to efficiently establish the database and localization. A good example is WiFiSLAM [9].

Similar to WiFiSLAM, SLAM based on the measurement of magnetic fields could also be implemented. Examples could be found in [10-12]. The process of the WiFiSLAM and magnetic SLAM is similar. Firstly a location is estimated. Then the estimated location is updated in the database. The updated database is used to estimate the further locations. The update of the database is usually achieved by interpolation. In [10-11], authors used a Gaussian process to interpolate the magnetic field and then a Rao-Blackwellized particle filter to determine the location. In [12], the focus is to solve the robot hijacking problem when using magnetic fields for SLAM. Same as [1012], particle filter is also the tool used for localization with the extra support from radio signals.

The mapping techniques have been evolved from spatial mapping to cognitive mapping, and inspiring biological map- 
ping. Spatial mapping, which is demonstrated in [9-12] is also the fundamental technique for mapping. With fine resolution of grid representations, spatial mapping is enough for accurate navigation task. However, large amount of data in database and use of only small amount of data for navigation causes inefficiency. Cognitive mapping was developed to resolve this program. One example is Hybrid Spatial Semantic Hierarchy [13], where experience/knowledge was considered in mapping.

However, the environment is dynamic and small change of the environment will cause great efforts for the spatial and cognitive mapping to adapt to the change. Inspired from the animal's ability to migrate or find home easily after completing a food hunting task, biological mapping was proposed to increase the robustness of mapping. Some great achievements have been made, as demonstrated in [14-15 ].

In this paper, the SLAM based on the measurement of the magnetic fields for indoor navigation is further investigated. It is called eSLAM: Simultaneous Localization and Mapping Based on Indoor Magnetic Fields. Although the process of magnetic SLAM is similar to WiFiSLAM, magnetic SLAM has its own characteristics. This is mainly because magnetic field is a vector quantity that has both magnitude and direction. In this paper, this main characteristic is considered and further measures are adopted to improve the localization accuracy and reduce the computation power. In addition, the particle filter applied in this paper is also improved to increase the accuracy of localization.

In summary, this paper has a few contributions:

1) When localization is performed, the three components of the magnetic field in three directions $\mathrm{x}, \mathrm{y}$, and $\mathrm{z}$ are used rather than the strength of the magnetic field. This increases the accuracy of localization.

2) When the database is updated, a Kriging interpolation method is used, where it has the ability to consider both the magnitude and angle.

3) When the particle filter is applied, the particle distribution is applied exponentially rather than Gaussian. The complexity of the particle filter is thus reduced and the convergence rate is increased. This is a closer approach to real-time implementation.

4) In order to deal with the measurement and system errors, a combined random noise and Gaussian white noise model is used in this paper. This can better describe the interference and achieve a more accurate result.

5) From the tests in an indoor environment, two interesting phenomena related to the particle filter have been discovered; one is the shift of location estimation after a sharp turning, the other is the accumulated errors, which requires further investigation to resolve the problems.

The rest of the paper is organized as follows. First the background and the objective of the research is introduced in Section II, including the assumptions of the research and limitations. Then the general method, procedure and details of localization based on particle filter and the improvements in particle filter are described in Section III. Following localization based on particle filter, the update of the database by Kriging interpolation is detailed in Section IV. After that simulations based on the described method are presented to evaluate the performance of the proposed method and realworld tests are undertaken to prove the effectiveness in Section V. Finally the paper is concluded in Section VI.

\section{RESEARCH BACKGROUND AND ASSUMPTIONS}

Indoor localization is becoming very hot recently because of the wide use of smartphones and application of location based services. Various technologies have been developed to address the localization problem, including optical, acoustic, radio and magnetic field. These technologies have their own advantages and disadvantages in terms of accuracy, coverage, cost, and real-time implementation.

Services based on location are also versatile, such as vehicle tracking in a car park [16], indoor object search [17], victim tracking in case of emergencies [18], and so on. Among these services, location based services in airport are particularly interested to the modern society because of the needs of daily life for travelling and business and also commercial values. In a large airport like Hartsfield-Jackson Atlanta Airport in the US, Beijing Capital Airport in China, Dubai Airport in United Arab Emirates, Heathrow Airport in London, Frankfurt Airport in Germany, thousands of passengers are served each year. In these large airports, to find the right location of service points, such as boarding gate, shops, restaurants, and toilets, is not an easy job. In order to make the people's life easier, various devices and applications have been developed. Among these devices and applications, a smart trolley was developed by working with industrial partner Wuxi Chigoo Interactive Technology Co. Ltd in China to help passengers navigate inside the airport and provide personalized services.

The picture of the smart trolley is shown in Fig.??. This is a combination of trolley and Android based tablet to provide information and media services. By using this smart trolley, passengers may carry their handbags, receive personalized flight information on the tablet, receive boarding reminding, enjoy the media and Internet services, and navigate themselves by the indoor location and navigation function. Current indoor location and navigation is accomplished by deploying a Radio Frequency Identification/Infrared Identification (RFID/IRID) network in the airport [19]. RFID/IRID can provide a relatively accurate estimation of the location. However, the limitations of RFID/IRID are obvious. First, this is a short-range radio technology and, therefore, a large number of RFID/IRID tags are required. The cost of tags and installation and maintenance costs are huge, particularly in labor expensive countries. Second, the installation of tags is impossible in some places, which makes the service of localization and navigation interrupted in some areas.

To overcome the limitations of the current RFID/IRID location and navigation technology, magnetic field based localization is considered because of the access without limitation and no installation of tags, where a magnetometer could be implemented in the tablet to locate an object. Similar to robotics is that the trolley is driven by wheels, where the distance of the trolleys traveled could be determined by available technologies, such as optical [20] and magnetic [21] rotation sensors. It is thus assumed that the distance of the trolley travelled is known in this paper. 


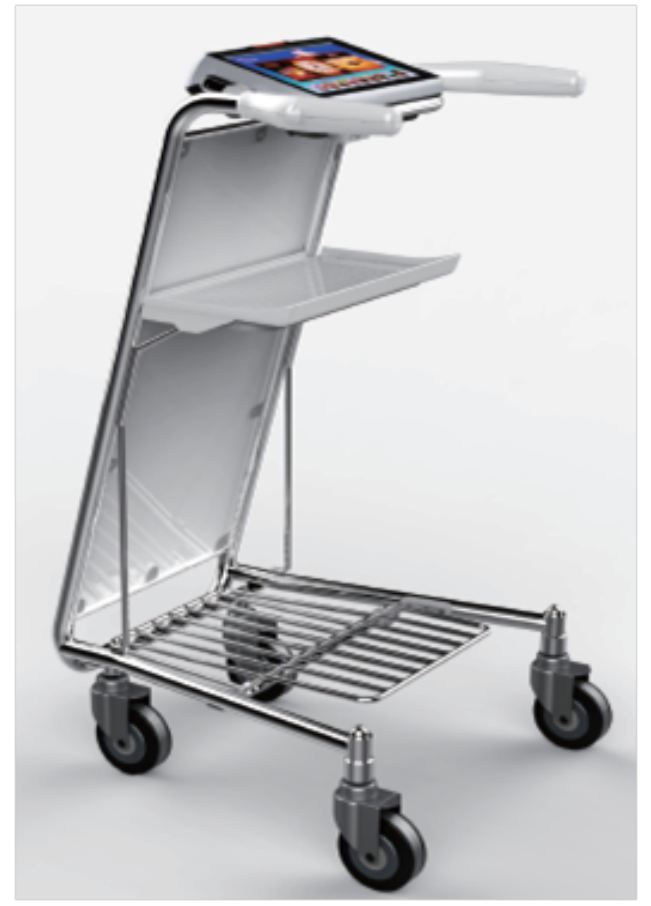

Fig. 1. Smart trolley used for airport services

\section{LOCALIZATION AND PARTICLE FILTER}

eSLAM is a process to make the localization and mapping conducted simultaneously. In this paper, it is targeting the localisation of the trolley. SLAM has two advantages. On the one hand, it may increase the accuracy of localization by using the developed map to reduce the location estimation errors based on the motion model; one the other hand, a more accurate map could be obtained by the positioning of the trolley.

In terms of localization, particle filter [22-23] is a popular mathematical tool for localization. Similar to [10-12], a RaoBalckwellized [23-24] particle filter is used to estimate the trajectories of the smart trolley and also the location map of the magnetic fields. The magnetic fields are then updated in the grid database by Kriging interpolation. The whole process is illustrated in Fig.?? by the flowchart. The details will be explained below.

Particle filter estimates the posterior of trajectories when the measurement and control information is available. The posterior of ESLAM is defined as:

$$
p\left(x_{t}, m \mid z_{1: t}, u_{t}\right)=p\left(m \mid x_{t}, z_{1: t}\right) p\left(x_{t} \mid z_{1: t}, u_{t}\right)
$$

where $x_{t}=\left[x_{v t}, y_{v t}, \Phi_{v t}\right]$ is the position and orientation of the trolley at time $t$, including the coordinate $\left(x_{v t}, y_{v t}\right)$ and angle $\Phi_{v t}, m=\left[x_{1}, y_{1}, x_{2}, y_{2}, \ldots, x_{n}, y_{n}\right]^{T}$ represents the location map of the magnetic fields, $z_{1: t}=\left[z_{1}, z_{2}, \ldots, z_{t}\right]^{T}$ is the series of measured magnetic fields to time $t, u_{t}=\left[l_{t}, \Phi_{t}\right]^{T}$ is the control information when the trolley is moved from time $t-1$ to $t$, where $l_{t}$ is the distance that trolley travelled from time $t-1$ to $t$ and $\Phi_{t}$ is the orientation of the trolley between the positions at $t-1$ and $t$. In this paper, the control information $l_{t}$ is $l_{t}=V_{t} * \Delta T$, where $V_{t}$ is the speed of the trolley, which

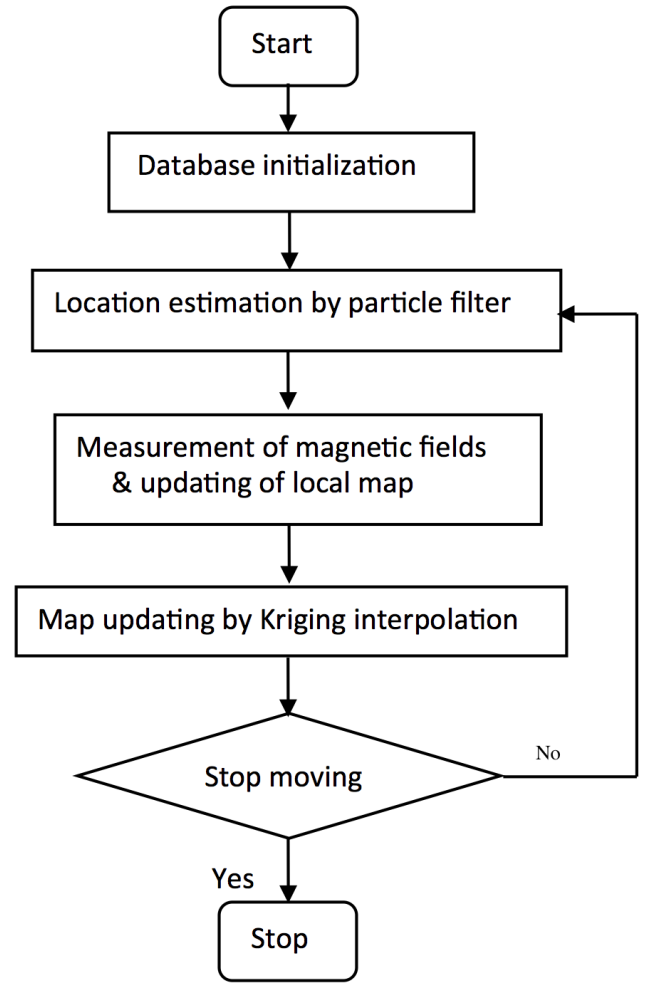

Fig. 2. Flowchart of eSLAM

could be obtained from the optical or magnetic sensors as described before, $\Delta T$ is the time step from $t-1$ to $t ; \Phi_{t}$ is calculated by the magnetic field components $\left(H_{x}, H_{y}\right)$. In order to calculate $\Phi_{t}$, firstly the trolley's direction is adjusted. Then the trolley is spun to get the maximum and minimum magnetic fields $X_{\max }, X_{\min }$ and $Y_{\max }, Y_{\min }$ in both $x$ and $y$ directions. After obtaining the maximum and minimum magnetic fields, a centre coordinate will be obtained by $\left\{\begin{array}{c}M i d x=\left(X_{\max }+X_{\min }\right) / 2 \\ M i d y=\left(Y_{\max }+Y_{\min }\right) / 2\end{array}\right.$. At last $\Phi_{t}$ is calculated by: $\Phi_{t}=\arctan ^{-1}\left(\left(H_{x}-M i d x\right) / H_{y}-M i d y\right)$.

The position of the trolley $p\left(x_{t} \mid z_{1: t}, u_{t}\right)$ is to be estimated. From the theory of particle filter, it is known that the position $x_{t}$ could be obtained from previous state $x_{t-1}$ and control information $u_{t}$, by (2):

$$
x_{t} \sim P\left(x_{t} \mid u_{t}, x_{t-1,} v_{t}\right)
$$

where control $u_{t}$ is obtained by other sensors, such as an optical sensor to measure the distance of the trolley traveled, $v_{t}$ is the error, including the measurement error caused by the sensor itself and also the environmental error. The correct modelling of these errors could improve the localisation accuracy [25]. In this paper, the errors are modeled by a mixture of random noise [26] and Gaussian white noise [10], where the distribution of the Gaussian white noise is $N(0,0.001)$. This value is obtained by the empirical evaluation. For each estimation of the location, there is an error between the estimated location and the real one. After deducting the random noise from the errors, the rest follow the Gaussian distribution. Because of relative steadiness of the geomagnetic field and the 
speed of the trolley and the convergence of the average power of Gaussian noise is 0 , therefore, the variance converges at about $0.001 \mathrm{dBW}$.

Because the distance of the trolley travelled and the orientation of the trolley are used for estimating the location, this method could be classified into dead-reckoning category. Similar to other dead-reckoning systems, errors accumulation, also called drift problem, is unavoidable. In [27], the problem was solved by integrating a RFID based local positioning system into the inertial measuring system to accurately determine a pedestrian's position. In [28], the errors from a compass were mitigated by allowing raw data to pass a low- pass filter. In this paper, experiments proved the existence of the accumulated errors.

Next the detailed steps of using particle filter for localization are to be explained.

\section{A. Initialization}

Supposing the initial position of the trolley, $X_{0}^{(i)}$, is known and the initial particles are uniformly distributed around the trolley. The weight of the initial particles is defined as $\omega_{0}^{(i)}(i=$ $1,2, \ldots, N)$, where $N$ is the total number of particles. In this paper $N=1000$. The size of the initial map is defined as $10 m \times 10 m$, based on the actual size of the area where the experiment was conducted. The initial value of the magnetic field in the grid map is set to a very small value, such as $1 \times 10^{-5}$.

\section{B. Resampling of particles}

According to the theory of Monte Carlo localization, the target location is estimated by random sampling. At time $t$, based on the particle set $X_{t-1}^{(i)}$ and control set $u_{t}$ and (2), the particles will be updated from $X_{t-1}^{(i)}$ to $X_{t}^{(i)}$. In this case, a new particle set $X_{t}^{(i)}$ at time $t$ will be obtained to estimate the location of the trolley at time $t$.

\section{Weight calculation of the particles}

The weight of the particles is calculated as:

$$
\omega_{k}^{(i)}=\omega_{k-1}^{(i)} * \frac{q\left(Z_{k} \mid X_{k}^{(i)}\right) q\left(X_{k}^{(i)} \mid X_{k-1}^{(i)}\right)}{P\left(X_{k}^{(i)} \mid X_{k-1}^{(i)}, Z_{k}, V_{k}\right)}
$$

where $P\left(X_{k}^{(i)} \mid X_{k-1}^{(i)}, Z_{k}, V_{k}\right)$ is the motion model (2) and $q(*)$ is posterior distribution.

Previous research by other researchers in localization by magnetic field [10-12, 29] has demonstrated that the characteristics of the map are described by the real magnetic field, with the strength $H=\sqrt{H_{x}^{2}+H_{y}^{2}+H_{z}^{2}}$ and direction of $<H_{x}, H_{y}, H_{z}>$. In this paper, the map is constructed by using the components of the magnetic field in three directions $H_{x}, H_{y}, H_{z}$, where three sub-maps are used. In this case, more characteristics of the map are used in localization, which increases the accuracy [30]. Because each particle represents one possible state of the object, the purpose of weighting is to assign a big weight value to the particle to represent the position to the real one and a small weight to the particle that brings a significant error. In this paper, the distribution of the weight is exponential, that is:

$$
\left\{\begin{array}{c}
D I S=\sqrt{\left|R \cdot m x_{t}-m x_{t}^{(i)}\right|+\left|R \cdot m y_{t}-m y_{t}^{(i)}\right|+\left|R \cdot m z_{t}-m z_{t}^{(i)}\right|} \\
\omega_{t}^{(i)}=\omega_{t}^{(i)} * \lambda * \exp ((-D I S) / \lambda)
\end{array}\right.
$$

where R.mx $x_{t}, R . m y_{t}, R . m z_{t}$ are the magnetic fields at time $t$ in $x, y, z$ directions, $H_{x}, H_{y}, H_{z}, m x_{t}^{(i)}, m y_{t}^{(i)}, m z_{t}^{(i)}$ are the corresponding magnetic fields in three directions stored in the map. The value of $\lambda$ can be determined based on the characteristics of the exponential distribution. When $\lambda$ is large, the distribution declines slowly and the sensitivity of the weight is low; when $\lambda$ is small, the distribution falls fast and the sensitivity of weight is high. In this paper, $\lambda$ is selected as 2 .

The complexity of the exponential distribution is $O(N)$, where $N$ is the number of particles. Comparing to the complexity of Gaussian distribution, which is $O\left(N^{2}\right)$ [22], it is simpler and thus faster in convergence. The accuracy of the localization will also be increased. The evaluation results could be found in Fig. 5.

\section{Normalize the weight}

Next step is to normalize the weight by (5):

$$
\omega_{k}^{i}=\omega_{k}^{(i)} / \sum_{i=1}^{N_{u m}} \omega_{k}^{(i)}
$$

\section{E. Calculate the effective particles and resampling}

Liu J. S. proposed a method to calculate the effective particles to estimate the posterior density from the current particles [31]. The equation is

$$
N_{e f f}=1 / \sum_{i=1}^{N}\left(\omega_{k}^{i}\right)^{2}
$$

A threshold $\delta$ is set. If the number of the effective particles is smaller than the threshold, then a resampling process is performed for the particle $X_{k}$ based on the weight $\omega_{k}^{(i)}$ [6][22]. The particles with lower weight values will be removed, while particles with higher weight value will be kept and used in next step.

\section{F. Location estimation of the smart trolley}

Based on the above procedures, the location of the trolley will be estimated and the measured magnetic field will be entered into the map. The map will be updated based on $p\left(m \mid x_{t}, z_{1: t}\right)$, when $m$ represents the map. Then the trolley is moved forward. It will repeat the process from step 2 until the trolley stops moving.

\section{MAP UPDATING BY KRIGING INTERPOLATION}

Kriging interpolation is a geo-statistical technique to estimate the points in unknown area. Its uniqueness is to estimate the point by weighting the neighbor points not only the 
distance but also the orientations. This makes it an ideal tool to interpolate the magnetic field because it is a vector quantity.

Kriging interpolation is somehow similar to the Inverse Distance Weighting (IDW) interpolation, where the closer of the known points to the estimated point the greater degree of the weight it will have. Its general form is shown in (7):

$$
\hat{Z}\left(S_{0}\right)=\sum_{i=1}^{N} \lambda_{i} Z\left(S_{i}\right), \sum_{i=1}^{N} \lambda_{i}=1
$$

where $Z\left(S_{i}\right)$ is the measurements, which are magnetic fields in this paper, at position $i, \lambda_{i}$ is the weight to $Z\left(S_{i}\right), S_{0}$ is the estimated position, $N$ is the number of known measured points, which is 100 in this paper. The target is to derive $\lambda_{i}$.

Kriging interpolation is generally classified into three categories, ordinary Kriging, simple Kriging and Universal Kriging. For ordinary Kriging method, weight $\lambda_{i}$ is decided by the distance between the known points and estimated point and also the relationship between the known measured points.

Among them, the coordinates of data points are marked by $S_{i}\left(a_{i}, b_{i}\right) i=1,2, \ldots, n$ in the grid map, and the coordinates of the points to be inserted are marked by $S_{0}\left(a_{0}, b_{0}\right)$. Then the distance between the scattered data points are to be found by:

$$
h_{i j}=\sqrt{\left(a_{i}-a_{j}\right)^{2}+\left(b_{i}-b_{j}\right)^{2}} i=1,2, \ldots, n ; j=1,2, \ldots, n
$$

The calculated distance values are arranged in ascending order and then the distance is divided into several groups, each containing a certain number of distance values. These values are combined into a distance group by:

$$
\left\{h_{m}^{\prime}\right\}=m \times \frac{\left(\max h_{i j}-\min h_{i j}\right)}{N_{H}}, m=1,2, \ldots, N_{H}
$$

where $N_{H}$ is the number of the distance group. According to (9), we can find the distribution characters of the semivariogram function:

$$
\gamma^{*}\left(h_{m}^{\prime}\right)=\frac{1}{2 N\left(h_{m}^{\prime}\right)} \sum_{i=1}^{N\left(h_{m}^{\prime}\right)}\left[Z\left(S_{i}\right)-Z\left(S_{i}-h_{m}^{\prime}\right)\right]^{2}
$$

where abscissa is $h_{m}^{\prime}$ and ordinate is $\gamma^{*}\left(h_{m}^{\prime}\right)$. Now we need to fit the semi-variogram function by an appropriate model function in order to produce a continuous curve to show the distribution of $\gamma^{*}\left(h_{m}^{\prime}\right)$. The weight is calculated by generating the empirical semi-variogram dataset and also a mathematical model, including spherical, exponential, Gaussian, power, Hole effect, quadratic, and rational quadratic (Rquadratic). From the experiments performed in this paper, it is found out that the spherical modeling gave us good interpolation results. Therefore, in this paper, the semi-variogram model used is:

$$
\gamma(h)=\left\{\begin{array}{cc}
c_{0}+c\left(1-\frac{2}{\pi} \cos ^{-1}\left(\frac{h}{\alpha}\right)\right)+\sqrt{1-\frac{h^{2}}{\alpha^{2}}} & 0<h<\alpha \\
c_{0}+c & h>\alpha \\
0 & \text { others }
\end{array}\right.
$$

where $c_{0}$ is nugget, $c$ is partial sill, $\alpha$ is codomain, and $h$ is active lag distance. In order to fit functions, we must find the model parameters $\left(c_{0}, c\right.$ and $\left.\alpha\right)$. Now we can obtain the expression of the variogram function $\gamma^{*}(h)$. It is easy to obtain the weight $\lambda_{i}$ by:

$$
\left\{\begin{array}{c}
\sum_{i=1}^{N} \gamma^{*}\left(S_{i}-S_{j}\right) \lambda_{i}-\mu=\gamma^{*}\left(S_{0}-S_{j}\right) j=1,2, \ldots, N \\
\sum_{i=1}^{N} \lambda_{i}=1
\end{array}\right.
$$

where $\mu$ is the Lagrange multipliers. Finally, according to (7), the predicted value of $\hat{Z}\left(S_{0}\right)$ is obtained. This interpolation method is used in the $5^{t h}$ step of the eSLAM described in Section III. The interpolated value will be added in the grids of the map, where a $10 \mathrm{~m}^{*} 10 \mathrm{~m}$ area with the grid resolution of $0.1235 \mathrm{~m} * 0.1235 \mathrm{~m}$ is used in this paper. After the interpolation is accomplished and local map is updated, the local map will be integrated into the whole map.

\section{ALGORITHMS EVALUATION AND EXPERIMENT}

In order to evaluate the effectiveness and performance of the proposed algorithms, evaluations are undertaken based on real experiments in an indoor environment. Fig. 3 shows the structure of an indoor exhibition area where furniture and separations are placed. Inside this area, a $10 \mathrm{~m} * 10 \mathrm{~m}$ square is selected for experiments. Magnetic fields are measured manually by the magnetometer implemented in the trolley. The magnetometer is Micromag3 designed and manufactured by PNI Sensor Corporation. Micromag3 is a magneto-inductive sensor where the inductance changes by $100 \%$ over its field measurement range. This property is used in designing a cost and space efficient ASIC. In addition, a PNI patented temperature and noise stabilized oscillator/counter circuit with a Serial Peripheral Interface (SPI) interface is incorporated in the design, where the SPI interface is compatible to microprocessor to make the measurement parameters and field measurement data accessible easily.

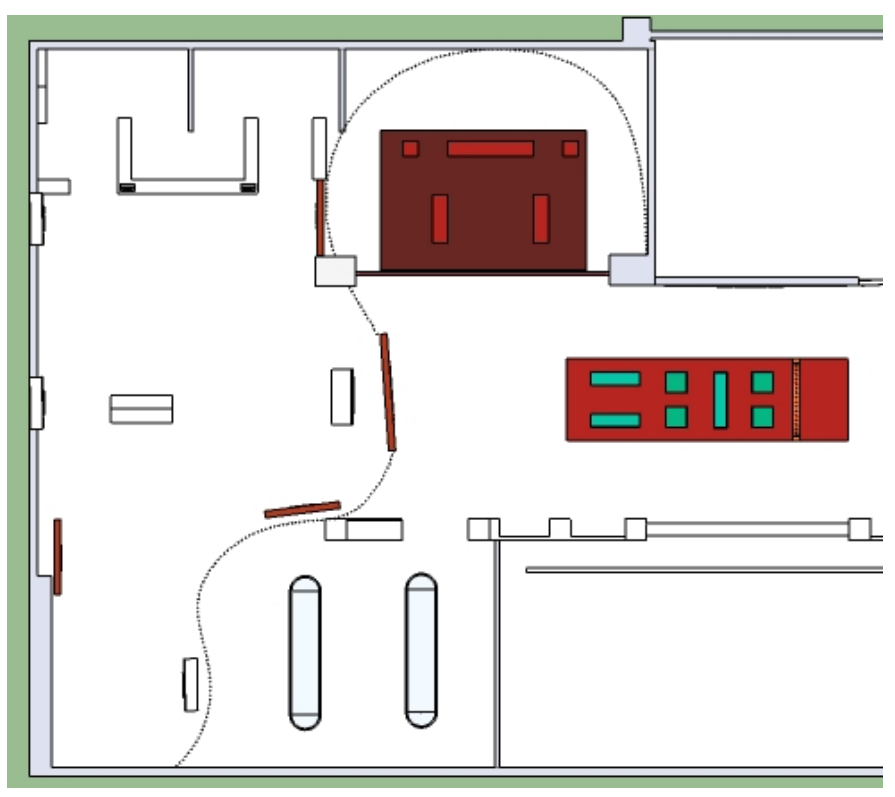

Fig. 3. An indoor area for tests

Magnetic fields are measured every $50 \mathrm{~cm}$ by the Micromag3 sensor. In order to ensure the accuracy of the measurement, an average of the measured magnetic fields is 
TABLE I. Performance of eSLAM

\begin{tabular}{ccccc}
\hline \hline Iteration times & $\begin{array}{c}\text { Average errors of distance } \\
\text { by method in }[6](\mathrm{m})\end{array}$ & $\begin{array}{c}\text { Average errors by method } \\
\text { in this paper }(\mathrm{m})\end{array}$ & $\begin{array}{c}\text { Calculation time by } \\
\text { method in [6] (s) }\end{array}$ & $\begin{array}{c}\text { Calculation time by } \\
\text { method in this paper }(\mathrm{s})\end{array}$ \\
\hline 5 & 6.3091 & 2.0854 & 9.731150 & 7.493352 \\
10 & 4.1332 & 1.3644 & 17.2427 & 12.6141 \\
15 & 3.8997 & 1.2708 & 24.7495 & 7.7471 \\
20 & 4.2494 & 1.7260 & 32.2855 & 22.9447 \\
\hline
\end{tabular}

obtained by measuring 10 times, with each lasting 2 minutes. The measurements are carried out continuously for 20 minutes, without any gap between each time and also without moving the meter. The measured data is recorded in a file and the average is taken every 2 minutes. The sampling rate is obtained by using the standard Android command $\left\{S E N S O R \_D E L A Y \_N O R M A L\right\}$ because the trolley and the smartphones used for the tests are Android operated. Fig. 4 shows an example of measured magnetic fields. From the figure, it can be seen that the magnetic fields have a clear difference in such a small area, which an electricity charging device is operating all the time on one side of the area and resulting high magnetic fields.

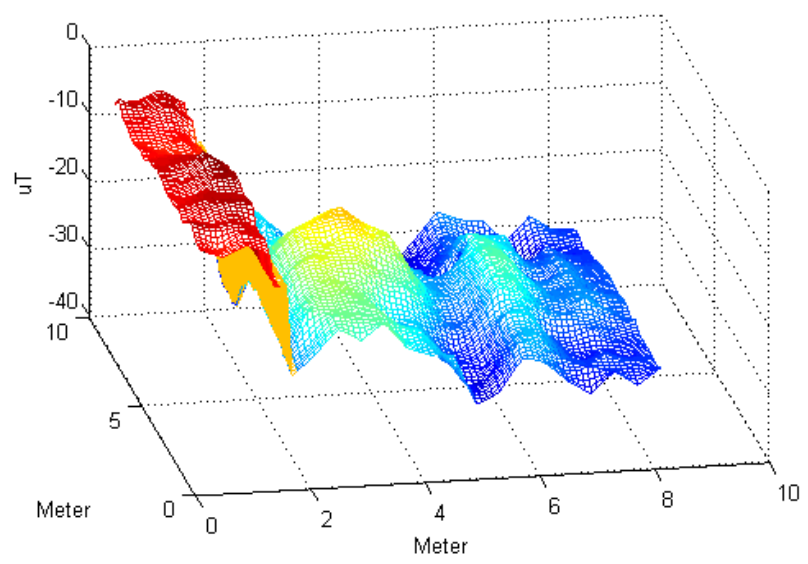

Fig. 4. Magnetic fields in the selected area

The measured data is interpolated and saved in a file as a magnetic field map for further evaluation of the proposed algorithm. The evaluation was carried out by two steps; the first step is to code the algorithm in Matlab for evaluating the performance and comparing the performance with other algorithms, the second step is to code the algorithm in C++ and implement it in an app for Android system. This app is installed on both the trolley and a smartphone to evaluate the effectiveness as a real-world application. By these two steps, the performance and the effectiveness of the algorithm could be fully justified.

Fig. 5 shows the results of localization on Matlab with different iteration times. Results on the left are from the algorithms proposed in this paper and the results on the right are from the method used in [6]. In the figure, data represented by + is the distribution of particles, square represents the real position and the circle represents the estimated position. It can be easily seen that the gap between the estimated location and real location is much smaller by using the proposed algorithm.
The results are also listed in Table I with calculation time. For example, when the particle filter was iterated for 15 times, the number of particles used in [6] is 1500 and the error between real location and estimated one is $3.8997 \mathrm{~m}$ and the time used to estimate is $24.7495 \mathrm{~s}$. However, in this paper, the number of the particles used is 1000 and the error is $1.2708 \mathrm{~m}$ and the time used to estimate is $17.7471 \mathrm{~s}$. This proves that the method proposed in this paper has a faster convergence rate and can achieve a more accurate result. It also proves that the more environmental properties used in the map, the more accurate is the localization.

From the table, it can also be seen that the calculation time to achieve a good result is still longer than the requirement of real-time applications. However, this could be compromised in practice by reducing the iteration times and number of particles.

The proposed algorithm is also evaluated by the app in the selected indoor environment. A smartphone with different magnetometer is used to test the generality of the developed algorithms. The model of the smartphone is Samsung Galaxy S3, where an AK8975C magnetometer is embedded. AK8975C is a product of Asahi Kasei Microdevices Corporation. It is a Hall sensor, where a self-test function is incorporated with internal magnetic source to confirm magnetic sensor operation. In addition, this device is worked by an on-chip oscillator so no external clock source is necessary. The video footage of testing by using the smartphone was uploaded on YouTube, where can be accessed at https://youtu.be/jwqPV2tv-gs.

The test results are shown in Fig. 6. During the tests, a fixed route was planned and indicated by + in the figure. A user carried a smartphone with app walking along the planned route. The estimated positions are also plotted on the map to see the difference. From the experiments, two important phenomena were discovered; one is the shift phenomenon, the other is accumulated error phenomenon.

As shown in the figure, after the second right angle from the starting point, estimated positions are shifted away from the real trajectory. It continues to shift away after the third right angle. The shifts occur later on after a sharp turn. After a final stop at 100 steps, which is equivalent to $50 \mathrm{~m}$, the difference between the estimated and real positions is $1.346 \mathrm{~m}$. The error rate is around $2.7 \%$, which is quite reasonable.

No previous evidence was found in the literature about the first phenomenon. However, we believe this is caused by the sharp turning. Further experiments were carried out to prove this phenomenon. In the experiments, the sharp turning was changed to curved turning and it can be seen that the shift doesn't exist anymore and the estimated positions fit the planned route very well. The test result is shown in Fig. 7. 


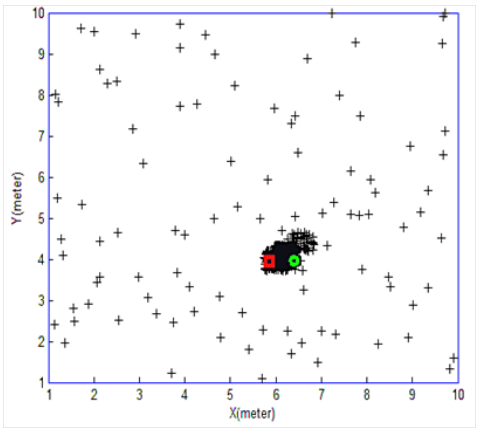

(a) 5 iterations with 1000 particles

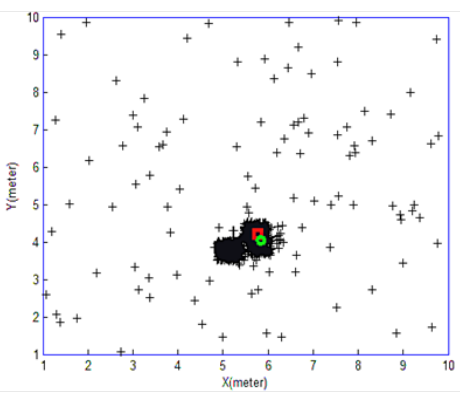

(c) 10 iterations with 1000 particles

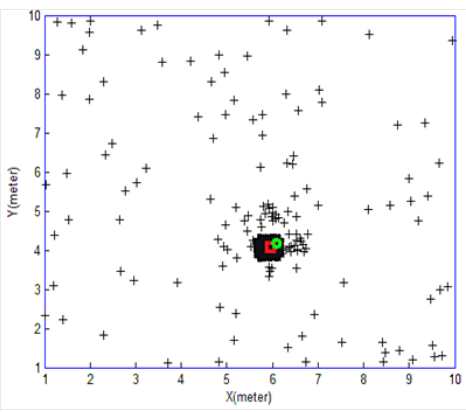

(e) 15 iterations with 1000 particles

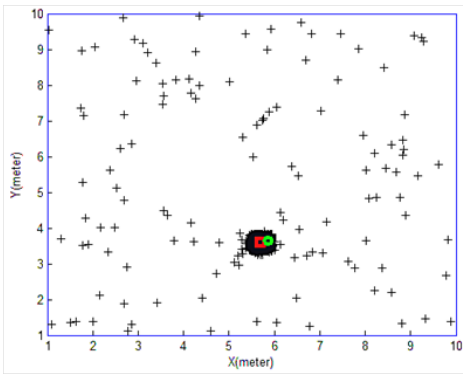

(g) 20 iterations with 1000 particles

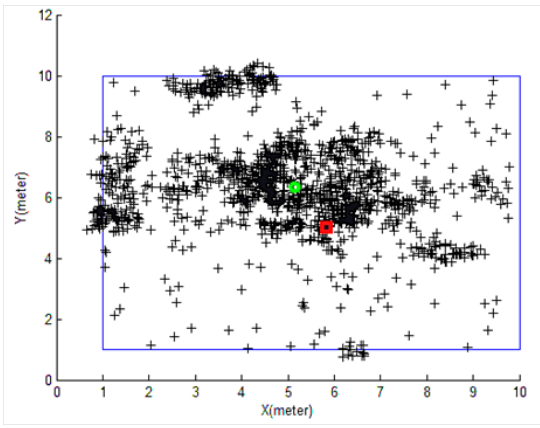

(b) 5 iterations with 1500 particles [6]

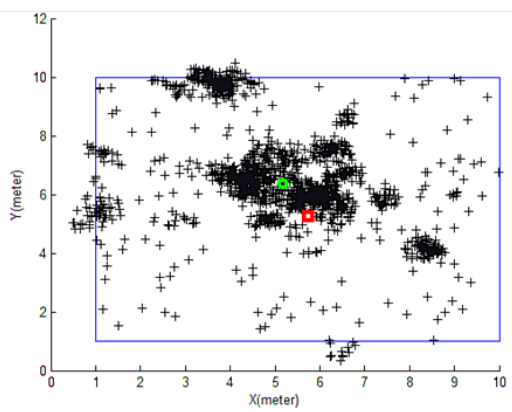

(d) 10 iterations with 1500 particles [6]

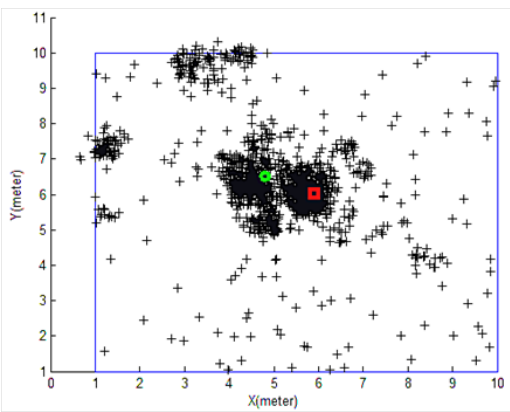

(f) 15 iterations with 1500 particles [6]

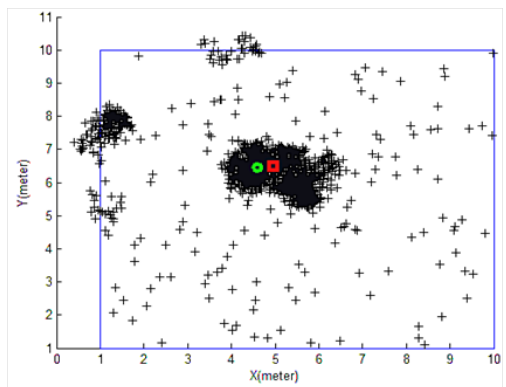

(h) 20 iterations with 1500 particles [6]

Fig. 5. Simulation results of eSLAM

There are two possible reasons for this. One is because of the movement of the body. After a sharp turning, the legs stop but the upper body and arms with smartphone being attached are still keeping moving a short distance because of inertia. This caused errors. Another possibility is because of the configuration of particle filter itself, where parameters are not optimally adjusted. However, in the future, further investigations are going to be undertaken regarding the speed of the movement and distance of movement after sharp turning, and looking into particle filter itself to find out the real reasons and resolve the problems.

From the experiments and simulations on Matlab, it is also discovered that the longer the route is, the higher the error is. There exist accumulated errors. Fig. 8 shows the size of the error with the steps of walking. At around 500 steps, the error could reach 5 meters. This might result in the failure of the algorithm and finally the failure of the method.

The phenomenon of accumulated errors has been discovered and investigated by other researchers, such as research in [28]. This is mainly because the number of effective particles is 


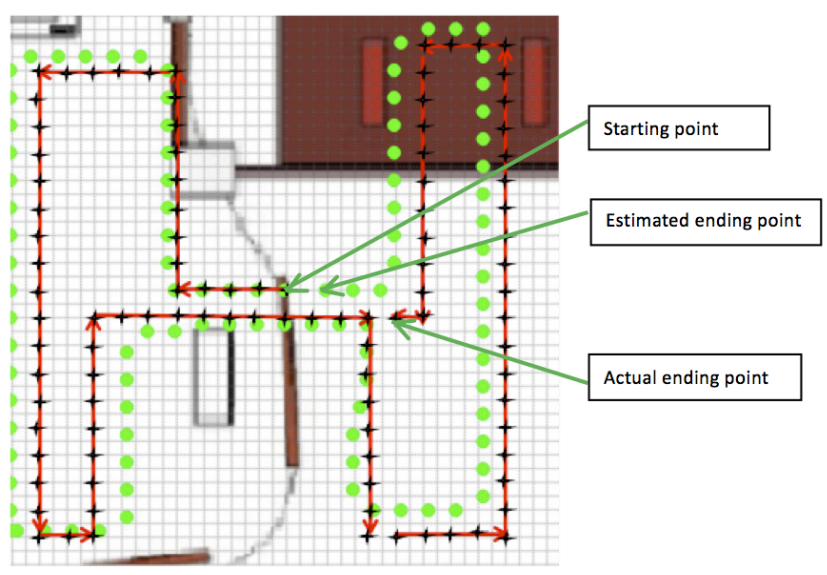

Fig. 6. Test result by an Android smartphone

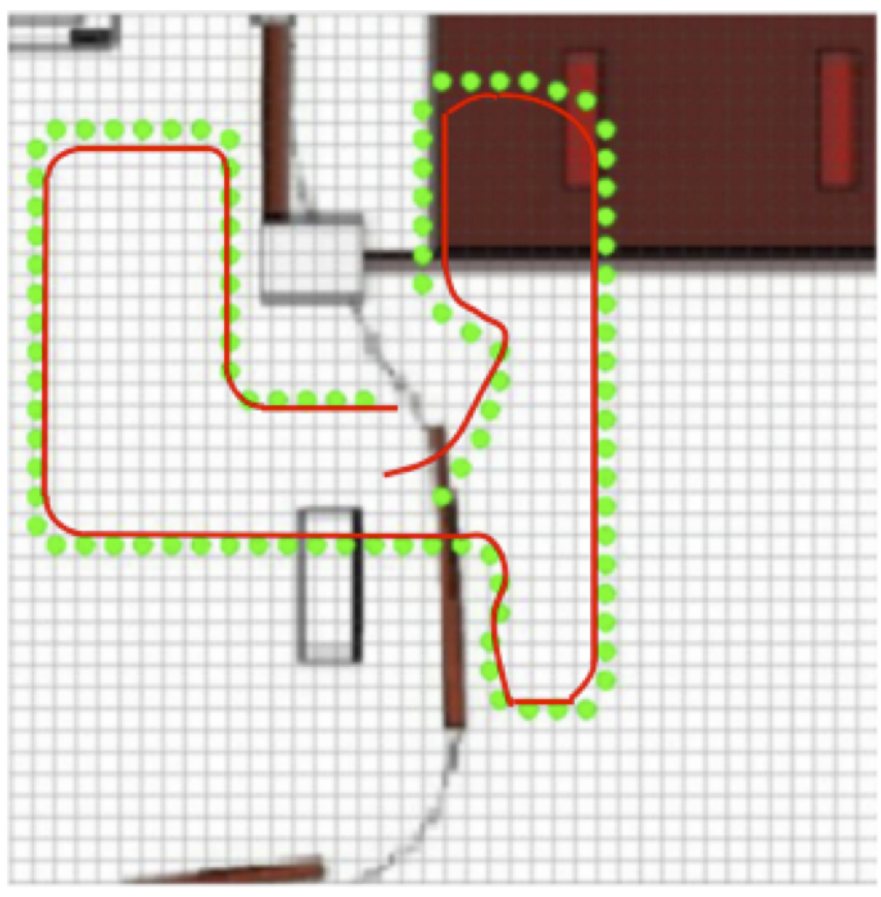

Fig. 7. Localization with curved route

reduced after iterations. Further research has also been carried out and a novel but simple evolutionary approach has been proposed to solve this problem. This will be reported in another paper.

The interpolation algorithm was also tested in the indoor environment. The distribution of the magnetic fields is shown in Fig. 9 and Fig. 10. They are the distribution of the magnetic field after the modeling and update after interpolation. Fig. 9 shows the magnetic fileds in $y$ direction. The results of the magnetic fields in $\mathrm{x}$ and $\mathrm{z}$ directions are similar. Fig. 10 shows the strength of the magnetic field. Figures on the left-hand side are the magnetic fields based on real measurements. On the right-hand side are the results of after interpolation. These are the maps after the trolley moving 500 steps. The blue color represents the areas with low magnetic fields, while the dark red color represents the strong magnetic fields. From the right-hand figures, it can see that the magnetic fields on the trajectories represent the original distribution of the magnetic fields. One problem found during the implementation

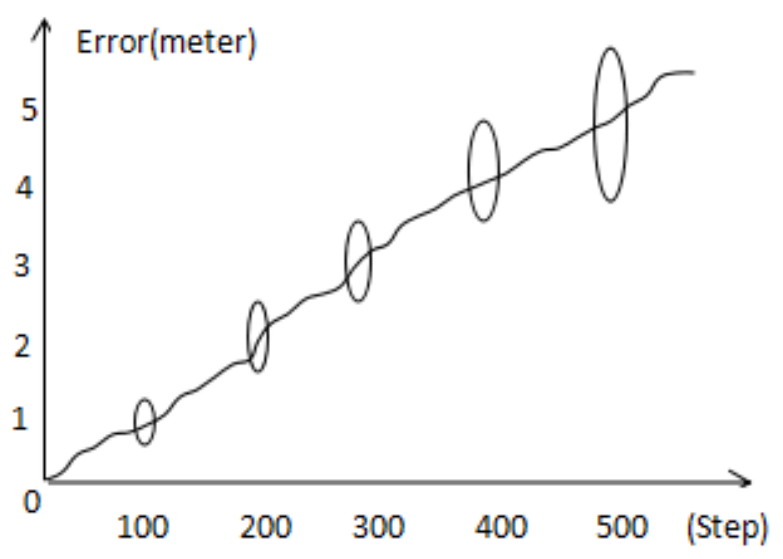

Fig. 8. Accumulated errors with walking distance

of interpolation process is that the battery drains quickly because of real-time updating and continuous exchange of data between the sensor and map. This needs to be resolved in the future to save the battery of the mobile devices. It also shows that the accuracy is increased on the trajectories with repeated movement of the trolley. Since the trolley is updating the map continuously, therefore in practice, if the magnetic fields have been changed because of the movement of the furniture or installment of new electronic devices, eSLAM shall correct the data in the map in real-time.

\section{CONCLUSIONS}

This paper presents a method to perform the eSLAM of a smart trolley in airport. Theoretical study, simulations on Matlab and real-world tests validated the proposed method. However, the experience learned from this work demonstrates the SLAM based on measurement of magnetic fields is still challenging. This can be shown in the following two aspects; one is the construction of the map, which is a really hard job for large-scale indoor environment. Further research work is required to make the map creation automated. The other is the implementation and application on smart device. Because of continuous data exchange between the map and localisation algorithm, it consumes a lot of power which drain the battery quickly. All these problems need to addressed in the future research work.

\section{REFERENCES}

[1] B, C. Larry and K. J. Lohmann, "True navigation and magnetic maps in spiny lobsters," Nature, vol. 421, no. 6918, pp. 60-63, 2003.

[2] N. F. Putman, K. J. Lohmann, E. M. Putman, T. P. Quinn, A. P. Klimley, and D. L.G. Noakes, "Evidence for geomagnetic imprinting as a homing mechanism in Pacific salmon," Current Biology, vol. 23, no. 4, pp. 312316, 2013.

[3] A. R. Holland and H. Barbara, "A strong magnetic pulse affects the precision of departure direction of naturally migrating adult but not juvenile birds," Journal of The Royal Society Interface, vol. 10, no. 81, pp. 2012-1047, 2013. 


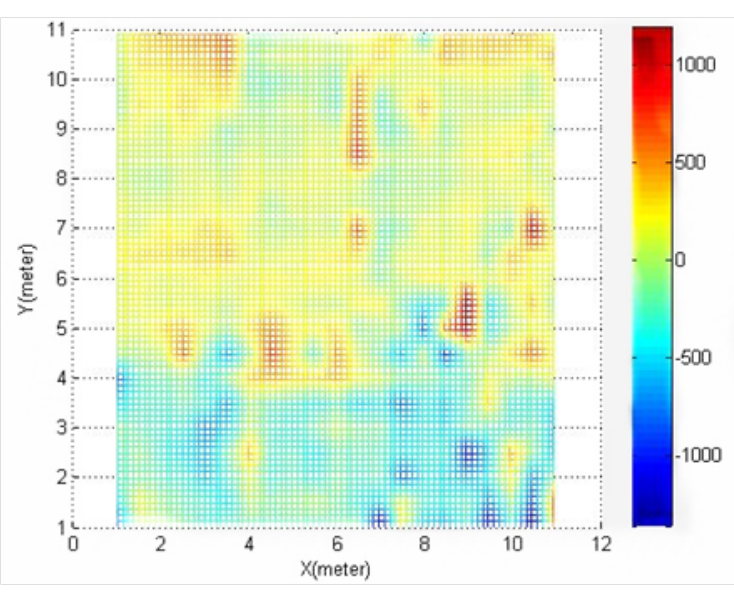

(a)

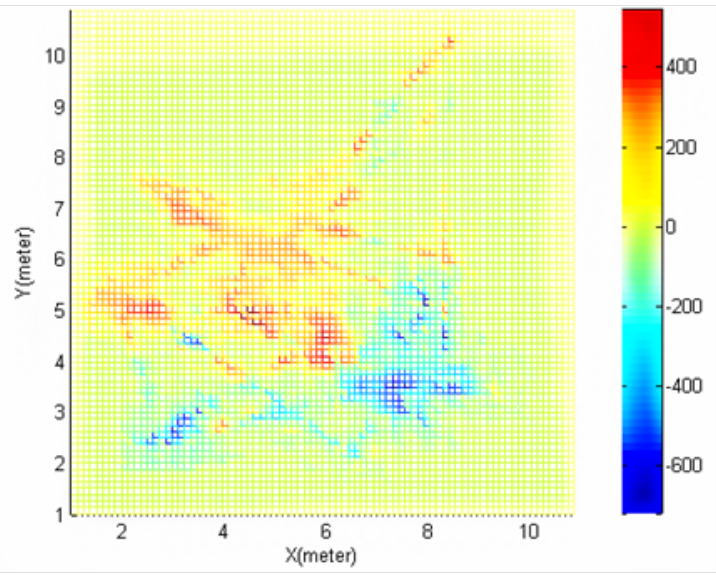

(b)

Fig. 9. Magnetic fields in y direction (left is the original distribution, right figure is the one after Kriging interpolation after 500 steps)

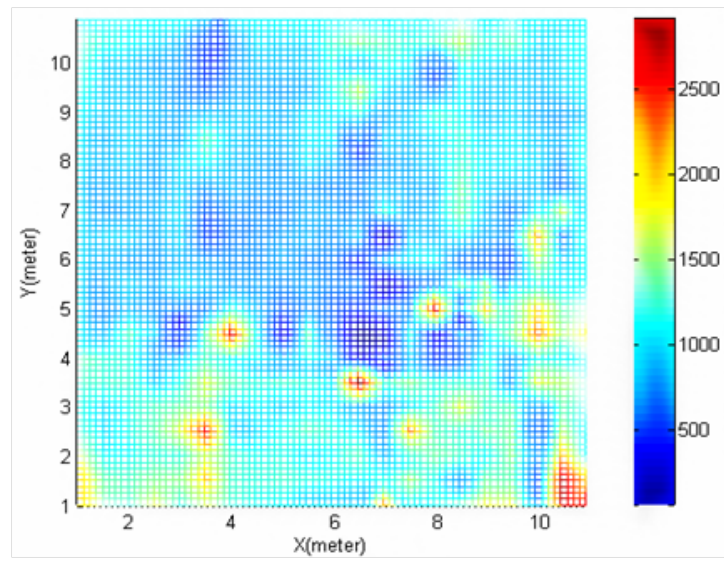

(a)

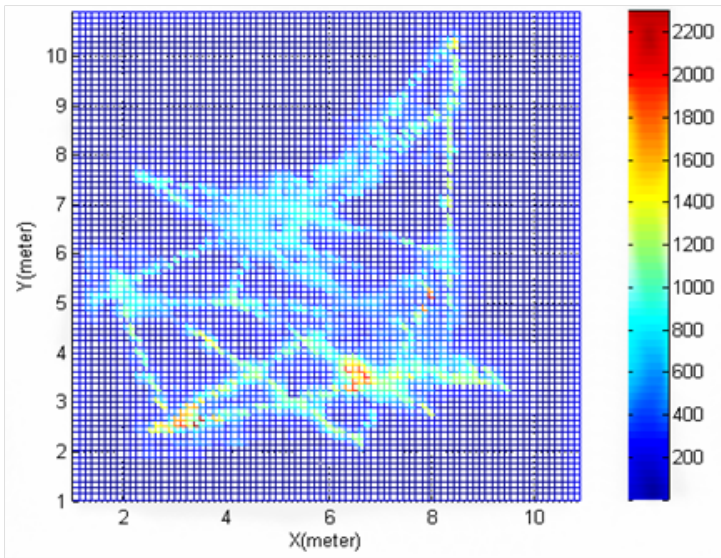

(b)

Fig. 10. Strength of the magnetic fields ((a) is the original, (b) is the one after Kring interpolation after 500 steps)

[4] B. Gozick, K. P. Subbu, R. Dantu, and T. Maeshiro, "Magnetic maps for indoor navigation," IEEE Trans. on Instrumentation and Measurement, vol.60, no.12, pp. 3883-3891, 2011.

[5] J. Haverinen and A.Kemppainen, "Global indoor selflocalization based on the ambient magnetic field," Robotics and Autonomous Systems, vol. 57, no. 10, pp. 1028-1035, 2009.

[6] X. H. Lu, Y. N. Dong, and X. H. Wang, "A Monte Carlo localization algorithm for 2-D indoor self-localization based on magnetic field," 8th International Conference on Communications and Networking in China(CHINACOM), Aug. 14-16, 2013, Guilin, China, pp. 563-568.

[7] A. Sheinker, B. Ginzburg, N. Salomonski, L. Frumkis and B. Z. Kaplan," "Localization in 2D Using Beacons of Low Frequency Magnetic Field,", IEEE Journal of Selected Topics in Applied Earth Observations and Remote Sensing, vol. 6, no. 2, pp. 1020-1030, April 2013.

[8] A. Sheinker, B. Ginzburg, N. Salomonski, L. Frumkis and B. Z. Kaplan, "Localization in 3-D Using Beacons of
Low Frequency Magnetic Field", IEEE Transactions on Instrumentation and Measurement, vol. 62, no. 12, pp. 3194-3201, Dec. 2013.

[9] B. Ferris, D. Fox, and N. Lawrence, "WiFi-SLAM using Gaussian process latent variable models," Proceedings of the 20th international joint conference on Artifical intelligence (IJCAI'07), Jan. 6-12, Hyderabad, India, pp. 2480-2485.

[10] I. Vallivaara, J. Haverinen, A. Kemppainen, and J. Roning, "Simultaneous localization and mapping using ambient magnetic field," 2010 IEEE Conference onMultisensor Fusion and Integration for Intelligent Systems (MFI), 5-7 Sept. 2010, Salt Lake City, UT, USA, pp. 14-19.

[11] I. Vallivaara,, J. Haverinen, A. Kemppainen, and J. Roning, "Magnetic field-based SLAM method for solving the localization problem in mobile robot floor-cleaning task," 2011 15th International Conference on Advanced Robotics (ICAR), 20-23 June 2011, Tallinn, Estonia, pp. 198-203.

[12] J. Jung, S. M. Lee and H. Myung, "Indoor Mobile Robot Localization and Mapping Based on Ambient Magnetic 
Fields and Aiding Radio Sources,", IEEE Transactions on Instrumentation and Measurement, vol. 64, no. 7, pp. 1922-1934, July 2015.

[13] B. Kuipers, and Y.T. Byun, "A Robot Exploration and Mapping Strategy Based on a Semantic Hierarchy of Spatial Representations," Robotics and Autonomous Systems, vol. 8, pp.47-63, 1991.

[14] S. X. Yang, A. Zhu, G. Yuan and M. Q. H. Meng,, "A Bioinspired Neurodynamics-Based Approach to Tracking Control of Mobile Robots," IEEE Transactions on Industrial Electronics, vol. 59, no. 8, pp.3211-3220, 2012.

[15] M. J. Milford and G. F. Wyeth, "Mapping a Suburb With a Single Camera Using a Biologically Inspired SLAM System," IEEE Transactions on Robotics, vol. 24, no. 5, pp. 1038-1053, Oct. 2008.

[16] J. Liu, R. Chen, Y. Chen, L. Pei, and L. Chen, "iParking: An Intelligent Indoor Location-Based Smartphone Parking Service", Sensors, vol.12, no.11, pp. Turkey, 51461214629, 2012

[17] B. Guo, S. Satake, and M. Imai, "Home-Explorer: ontology-based physical artifact search and hidden object detection system," Mobile Information Systems, vol. 4, no.2, pp. 81-103, 2008.

[18] R. Zhang, F. Hoflinger and L. Reindl, "Inertial Sensor Based Indoor Localization and Monitoring System for Emergency Responders", IEEE Sensors Journal, vol. 13, no. 2, pp. 838-848, Feb. 2013.

[19] ID identification apparatus and identification method as well as using method, by W. Lan, H. Zhou, C. Pan, C. Zheng, and T. Chen. (2008, April 10). China patent CN101276403A, CN101276403B,WO2009124506A1[Online]: Available: http://www.google.com.bz/patents/CN101276403B?cl=en

[20] Fiber optic rotation sensor Bergh, by A. Ralph, H.C. Lefevre, and H. J. Shaw. (1983, Oct. 18). U.S. Patent No. 4410275.

[21] Magnetic rotational position sensor with improved output linearity, by R. H. Luetzow. (1995, Aug. 22). U.S. Patent No. 5444369[Online]: Available: http://www.google.co.uk/patents/US5444369

[22] M. Montemerlo, S. Thrun, D. Koller and B. Wegbreit, "FastSLAM: A factored solution to the simultaneous localization and mapping problem," AAAI National Conference on Artificial Intelligence, Menlo Park, CA, USA, July 28 - August 1, 2002, pp. 593-598.

[23] I. M. Rekleitis, "A particle filter tutorial for mobile robot localization," Centre for Intelligent Machines, McGill University, Tech. Rep. TR-CIM-04-02, 2004.

[24] Widyawan, M. Klepal, S. Beauregard, and D. Resch, "A novel backtracking particle filter for pattern matching indoor localization," Proceedings of the first ACM international workshop on Mobile entity localization and tracking in GPS-less environments, San Francisco, California, USA, September 14 - 19, 2008, pp. 79-84.

[25] W. Suski, S. Banerjee, and A. Hoover, "Using a Map of Measurement Noise to Improve UWB Indoor Position Tracking," IEEE Trans. on Instrumentation and Measurement, vol. 62, no. 8, pp. 2228-2236, 2013.
[26] C. M. Wang, "Location estimation and uncertainty analysis for mobile robots," IEEE International Conference on Robotics and Automation, Philadelphia, PA, USA, 24-29 Apr 1988, pp. 1231-1235.

[27] A. Jiménez, F. Seco Granja, J. C. Prieto Honorato, and J. Guevara Rosas, "Accurate Pedestrian Indoor Navigation by Tightly Coupling a Foot-mounted IMU and RFID Measurements," IEEE Trans. on Instrumentation and Measurement, vol. 61, no. 1, pp. 178-189, 2012.

[28] L. Fang et al., "Design of a wireless assisted pedestrian dead reckoning system-The NavMote experience," IEEE Trans. on Instrumentation and Measurement, vol. 54, no. 6, pp. 2342-2358, 2005.

[29] O. Gromenko, P. Kokoszka , L. Zhu, and J. Sojka, "Estimation and testing for spatially indexed curves with application to ionospheric and magnetic field trends," The Annals of Applied Statistics, vol.6, no.2, pp. 669-696, 2012.

[30] B. H. Li, H. Gallagher, A. G. Dempster, and C. Rizos, "How feasible is the use of magnetic field alone for indoor positioning", 2012 International Conference on Indoor Positioning and Indoor Navigation (IPIN), Sydney, Australia, 2012, pp. 1-9.

[31] J. S. Liu, "Metropolized independent sampling with comparisons to rejection sampling and importance sampling," Statistics and Computing, vol.6, no.2, pp.113-119, 1996. B. N. Oreshkin and M. J. Coates, "Analysis of error propagation in particle filtersg", Annals of Applied Probability, vol. 21, no.6, pp.2343-2378, 2011. 